\title{
Aplicación del aprendizaje basado en problemas en el programa de Ingeniería Industrial: caso de estudio aplicado en el curso de Gestión de Cadenas de Suministro
}

\author{
Application of Problem-Based Learning in the Industrial Engineering program: Case \\ Study Applied in the Supply Chain Management Course
}

Aplicação da aprendizagem baseada em problemas no programa de Engenharia Industrial: estudo de caso aplicado no curso de Gestão da Cadeia de Abastecimento

\author{
José Ruiz-Meza ${ }^{1}$ \\ Mariel Castellanos-Adarme ${ }^{2}$ \\ Faber Alzate-Ortiz ${ }^{3}$ (i) \\ Alfredo Flórez-Gutiérrez ${ }^{4}$ (D)
}

Recibido: noviembre de 2020

Aceptado: marzo de 2021

Para citar este artículo: Ruiz-Meza, J., Castellanos-Adarme, M., Alzate-Ortiz, F. y Flórez-Gutiérrez, A. (2021). Aplicación del aprendizaje basado en problemas en el programa de Ingeniería Industrial: caso de estudio aplicado en el curso de Gestión de Cadenas de Suministro. Revista Científica, 41(2), 169-183.

https://doi.org/10.14483/23448350.16248

\section{Resumen}

El aprendizaje basado en problemas (ABP) es una estrategia didáctica orientada al enfoque por competencias dentro de las teorías constructivistas. El ABP genera en el estudiante un proceso de aprendizaje colaborativo, autoaprendizaje y aprendizaje significativo a partir de una situación problema contextualizada. Este tipo de metodología surge como respuesta de apoyo al proceso del cambio pedagógico que se da bajo el enfoque por competencias en la educación superior para generar un aprendizaje activo en los estudiantes. En este trabajo se desarrolló una metodología de cinco fases (identificación de situación problema, organización del trabajo para el aprendizaje colaborativo, seguimiento y apoyo al autoaprendizaje, construcción de soluciones aplicadas, y evaluaciones y retroalimentaciones) para aplicar el ABP en el curso de Gestión de Cadenas de Suministro dentro del programa de Ingeniería Industrial de la Corporación Universitaria del Caribe CECAR. Se realizó una revisión sistemática de literatura que identifica la baja tasa de aplicación de la metodología en la ingeniería industrial. Para la aplicación de la estrategia, se tomaron como base tres problemas contextualizados asociados a empresas de los sectores económicos que tienen mayor influencia en

1. Corporación Universitaria del Caribe. Sincelejo, Colombia. jose.ruizm@cecar.edu.co.

2. Corporación Universitaria del Caribe. Sincelejo, Colombia. mariel.castellanos@cecar.edu.co.

3. Universidad San Buenaventura. Medellín, Colombia. viceacademica@usbmed.edu.co.

4. Corporación Universitaria del Caribe. Sincelejo, Colombia. alfredo.florez@cecar.edu.co. 
la generación del Producto Interno Bruto (PIB) de la región. El impacto académico en un total de cuatro grupos fue evaluado. Adicionalmente, se alcanzaron las competencias, los desempeños y los productos esperados en los cursos, obteniendo así una gran aceptación y eficiencia en la aplicación del ABP.

Palabras clave: aprendizaje basado en problemas; competencias, desempeños; ingeniería industrial; problema contextualizado.

\section{Abstract}

Problem-based learning (PBL) is a didactic strategy oriented to the competency-based approach within constructivist theories. PBL generates a collaborative learning process in the student, as well as self-learning and meaningful learning from a contextualized problem situation. This methodology arises to support the process of pedagogical change that occurs under the competency-based approach in higher education in order to generate active learning in students. In this paper, a five-phase methodology was developed (identification of the problem situation, organization of the work for collaborative learning, monitoring and support for self-learning, construction of applied solutions, and evaluations and feedback) to apply PBL in the Supply Chain Management course of the Industrial Engineering Program at Corporación Universitaria del Caribe (CECAR). A systematic literature review was carried out to identify the low application rate of the methodology in the field of industrial engineering. For the application of the strategy, three contextualized problems were taken as a basis, which are associated with companies of the economic sectors that have the highest influence in the generation of the Gross Domestic Product (GDP) of the region. The academic impact on a total of four groups was evaluated. Additionally, the expected competences, performances, and products in the courses were achieved, thus obtaining a great acceptance and efficiency in PBL application.

Keywords: competences; contextualized problem; industrial engineering; performance; problem-based learning.

\section{Resumo}

A aprendizagem baseada em problemas (PBL) é uma estratégia didática orientada para a abordagem baseada na competência no âmbito das teorias construtivistas. PBL gera no aluno um processo de aprendizagem colaborativa, autoaprendizagem e aprendizagem significativa a partir de uma situação problemática contextualizada. Este tipo de metodologia surge como uma resposta para apoiar o processo de mudança pedagógica que ocorre no âmbito da abordagem baseada na competência no ensino superior com o intuito de gerar aprendizagem ativa nos estudantes. Neste trabaIho, foi desenvolvida uma metodologia de cinco fases (identificação da situação problemática, organização do trabalho para a aprendizagem colaborativa, monitorização e apoio à autoaprendizagem, construção de soluções aplicadas, e avaliações e feedback) para aplicar PBL no curso de Gestão da Cadeia de Abastecimento do programa de Engenharia Industrial da Corporación Universitaria del Caribe CECAR. Foi realizada uma revisão sistemática da literatura para identificar a baixa taxa de aplicação da metodologia na engenharia industrial. Para a aplicação da estratégia, foram tomados como base três problemas contextualizados associados a empresas dos setores econômicos que têm maior influência na geração do Produto Interno Bruto (PIB) da região. O impacto acadêmico em um total de quatro grupos foi avaliado. Adicionalmente, as competências, performances e produtos esperados nos cursos foram alcançados, obtendo assim uma grande aceitação e eficiência na aplicação do PBL.

Palavras-chaves: aprendizagem baseada em problemas; competências; desempenho; engenharia industrial; problema contextualizado.

\section{Introducción}

El cambio pedagógico orientado hacia el aprendizaje por competencias, empleado para fomentar el aprendizaje activo, sugiriere un reto para la educación superior (Moust, Berkel, y Schmidt, 2005; Wedel, Müller, Pfetsch e Ittel, 2019). Este cambio va de la mano con las exigencias del mercado para los nuevos profesionales que requieren un enfoque de aprendizaje diferente, que permita mejorar las habilidades y competencias para resolver situaciones problemas del mundo laboral (Andersen, 
Brunoe y Nielsen, 2019). En este sentido, la metodología de aprendizaje basado en problemas (ABP) fundamentada en las teorías cognitivas (Dolmans, Wolfhagen, van der Vleuten y Wijnen, 2001; Moust et al., 2005; Rovers, Clarebout, Savelberg y van Merriënboer, 2018), supone identificar un problema contextual que permita al estudiante la construcción de su propio conocimiento a partir del ejercicio de generar una solución o soluciones eficientes (Soares et al., 2013). Con ello se da un cambio sustancial, de la metodología tradicional memorística a un nuevo enfoque constructivista. Lo que sugiere cambiar la enseñanza basada en clases magistrales y la resolución de problemas tipificados y alejados de las necesidades de la sociedad, por una nueva metodología activa (Parra, Amariles y Castro, 2016).

Este cambio fomenta el mejoramiento del aprendizaje y la práctica profesional en cuanto a la adquisición de competencias duras y blandas, de manera que el estudiante esté alineado con el contexto laboral que lo espera (Könings, BrandGruwel y van Merriënboer, 2005; Rovers et al., 2018). Adicionalmente, se priorizan los procesos de autoaprendizaje a través de problemas contextualizados y significativos (Herrán y Vega, 2006); lo cual concluye en una serie de cambios que van ligados a la realización de un proceso de enseñanza-aprendizaje flexible, que se desarrolla desde la praxis con un carácter participativo, activo, interdisciplinario y con base en la investigación (Betancourt, 2006).

La orientación en el enfoque basado en competencias y el horizonte pedagógico de la Corporación Universitaria del Caribe CECAR hacia el modelo sociocognitivo apoya el uso de este tipo de metodologías activas. Con base en esto, se pretende la restructuración y actualización de los syllabus, aplicación de nuevas herramientas de enseñanza y cualificación de docentes ante las nuevas tendencias de la educación. De modo que el $\mathrm{ABP}$ se muestra como una herramienta didáctica que aporta significativamente en el logro de tres puntos relevantes: i. alcanzar las competencias establecidas dentro de los sistemas de competencias de cada programa; ii. transformar al docente en un orientador; iii. generar focos de problemas reales que serán trabajados por pequeños grupos autodirigidos y centrados solo en el estudiante para mejorar el proceso educativo (Barrows, 1996; Rovers et al., 2018).

Ahora bien, la formación de ingenieros industriales cecarenses de calidad supone una orientación desde la propuesta misional del programa para que el estudiante adquiera "competencias en el diseño, desarrollo, implementación y mejora de sistemas que integren personas, materiales, información, equipos y energía" (Programa de Ingeniería Industrial, 2018); para lo cual los proyectos de aula basados en ABP que se generan en cada curso, desde primer hasta último semestre, son una herramienta para dar soluciones eficientes a problemas del contexto real de la región y que apunten al alcance de estas competencias en el estudiante.

En este trabajo se desarrollan cinco fases para la aplicación de la metodología ABP, las cuales son: i. identificación o diseño de situaciones problemas; ii. organización del equipo para el aprendizaje colaborativo; iii. seguimiento y apoyo al autoaprendizaje; iv. construcción de soluciones aplicadas; v. evaluación y retroalimentación. Se toma como caso de estudio el curso de Gestión de Cadenas de Suministro y se aplica la metodología de ABP identificando tres problemas clave dentro de los principales sectores económicos que aportan el $44.9 \%$ PIB del departamento de Sucre (comercio, hoteles y reparación $18.5 \%$, construcción $10.4 \%$, agricultura, ganadería y pesca $9.3 \%$, industrias manufactureras $6.7 \%$ ) (Mincomercio, 2019). Posteriormente, se desarrollan soluciones para los problemas dentro del marco de la metodología de cinco fases para el ABP.

El resto de este trabajo está estructurado de la siguiente manera: en la sección 2 se realiza una breve revisión sistemática de la literatura sobre el ABP y su aplicación en la educación en ingeniería industrial; en la sección 3 se describen los 
materiales y métodos aplicados en el estudio; en la sección 4 se presentan los resultados; finalmente, la sección 5 corresponde a las discusiones, conclusiones y recomendaciones.

\section{Aplicación del ABP en la ingeniería industrial}

Para realizar una breve revisión sistemática de la aplicación del ABP en la educación de la ingeniería industrial, se define la ecuación de búsqueda: "problem-based learning" e "industrial engineering", la cual fue aplicada en las bases de datos Dialnet, Redalyc, Scopus y Science Direct. Se obtuvo un total de 129 documentos a los cuales se les aplican criterios de exclusión en cuanto a: i. consideración de solo artículos científicos y artículos de revisión, ii. documentos en idioma inglés y español, iii. exclusión por duplicidad, iv. revisión de títulos y resúmenes acordes con el objetivo de la revisión; con lo cual se excluyeron 116 documentos para obtener un total de 13 documentos potenciales. El número de documentos a lo largo del tiempo se muestra en la Figura 1.

En nuestra revisión encontramos el trabajo realizado por Inelmen (2003), quien establece la importancia y las ventajas de la aplicación del ABP dentro del currículo de las ingenierías, entre ellas la ingeniería industrial. Estas ventajas se reflejan en los estudiantes y el docente como punto de partida para que este último realice de manera consecuente sus propias investigaciones, reconociendo el trabajo colaborativo con los estudiantes. Por otra parte, Lau y Mak (2004), Lau y Mak (2005) y Lau, Mak y Ma (2006) emplean el ABP mediante la resolución de dos problemas contextualizados de dos empresas virtuales, a través de una nueva plataforma que permite la interacción del estudiante con el problema. Adicionalmente, permite el cargue de información y verificar así la dinámica del impacto de las actividades que realizan para solucionarlo. Este sistema es denominado sistema multimedia interactivo de e-learning (IMELS) y es implementado para mejorar el proceso de enseñanza-aprendizaje de los estudiantes del departamento de Ingeniería Industrial de la Universidad de Hong Kong.

Mesa, Álvarez, Villanueva y de Cos (2008) aplican el ABP en el curso de dirección de proyectos de ingeniería del programa de Ingeniería Industrial perteneciente a la Escuela Politécnica Superior de Ingeniería de Gijón (EPSIG) de la Universidad de Oviedo, ubicada en España. La aplicación del ABP se realiza de manera articulada con las tecnologías de la información y las comunicaciones (TIC), evidenciando la importancia del trabajo grupal a pesar de la no presencialidad. Echavarria (2010) aplica el ABP en el curso de gestión de procesos

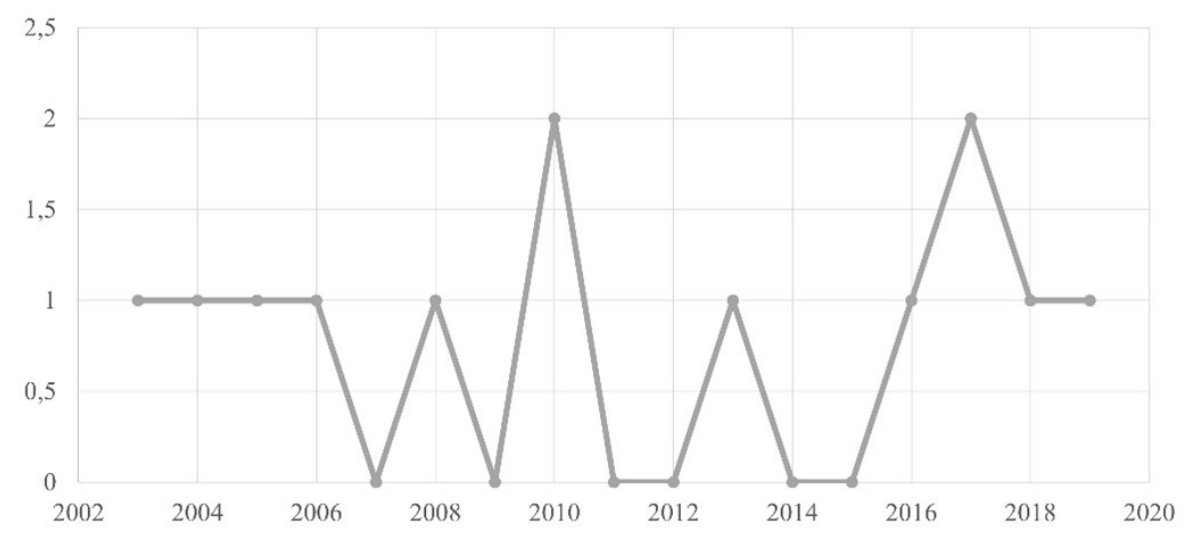

Figura 1. Número de documentos por año de publicación.

Fuente: elaboración propia. 
de la Escuela de Ingeniería de Antioquia durante cinco semestres consecutivos para un total de 118 estudiantes. La aplicación se realiza en nueve métodos de evaluación: presentación grupal, presentación individual, caso basado en reportes individuales, portafolios, "triple salto", autoevaluación, evaluación y retroalimentación por parte de los compañeros, informes de reflexión y, finalmente, examen basado en el problema. La aplicación continua permitió realizar mejoras al proceso y aumentar el nivel de aprendizaje gracias a la interacción social y al contexto basado en la aplicación práctica.

Los autores Ostojic et al. (2010) desarrollan un conjunto de didácticas de trabajo para solucionar problemas reales identificados por estudiantes de mecatrónica e ingeniería industrial de la Universidad de Novi Sad en Serbia. Se emplean los equipos, las herramientas del laboratorio de mecatrónica y los conocimientos previos adquiridos por los estudiantes en los diferentes cursos realizados. Por otra parte, para la mejora del proceso educativo en la Universidad Católica de la Santísima Concepción de Chile, Muñoz, Martínez, Cárdenas y Cepeda (2013) implementan una restructuración curricular que permite un aprendizaje activo tomando como metodologías de aprendizaje enfoques como el ABP. Se realiza una nueva planificación curricular para los estudiantes en el curso de introducción a la ingeniería industrial, que les permite solucionar pequeños problemas con base en los diferentes tópicos que son trabajados durante la clase.

Nande-Betancurt (2016) aplica una situación problema para evaluar la incursión del ABP en el curso de gestión estratégica y comercial de la Filial de Ciencias Técnicas Norte de la Universidad Tecnológica de La Habana "José Antonio Echeverría". Los resultados demuestran que se pueden mejorar los resultados académicos del curso al incluir de manera gradual el uso de esta metodología. Otro aporte fue el realizado por Baykasoğlu, Demirkol Akyol y Demirkan (2017), quienes toman como base un problema real de análisis de riesgo ergonómico para ser solucionado por los estudiantes de ingeniería industrial de la Universidad de Dokuz Eylül (Turquía). Se utiliza una herramienta desarrollada en Excel que toma como base la evaluación ergonómica de tres métodos y compara los resultados.

Tortorella y Cauchick-Miguel (2017) proponen un enfoque de trabajo basado en ABP para solucionar problemas con Lean Manufacturing (LM). Con este enfoque se logra un trabajo articulado entre la teoría de LM y los aspectos prácticos que conlleva trabajar en un problema o problemas contextualizados. Adicionalmente, se desarrollan en los estudiantes habilidades y competencias como aprendices independientes que los hacen más apetecibles en el mercado laboral. Otros autores como Decker-Lang (2018) aplican el ABP para solucionar problemas de administración en cuanto a la evaluación cultural y económica de museos públicos. Con esto, los estudiantes tomaron los problemas de estos organismos gubernamentales y brindaron enfoques de solución con base en todo lo aprendido a lo largo de la carrera y del curso. Esta aplicación generó impacto dentro y fuera de la institución, interés por la ciencia y proyectos que permiten el trabajo colaborativo de los estudiantes.

Finalmente, en el trabajo desarrollado por Martínez-León, (2019) se describe la aplicación del ABP para desarrollar competencias en estudiantes en el marco de la solución de problemas en articulación universidad-empresa bajo un enfoque de aplicación de Lean Six Sigma (LSS). Con este trabajo se realiza un cambio en el modo tradicional de educación basado en actividades, lecturas, casos de estudio, entre otros aspectos, y se genera una metodología nueva de trabajo con base en seis fases: explorar, agregar, reconocer vacíos, comenzar, ejecutar, realizar y recompensar.

\section{Otras metodologías aplicadas en la ingeniería industrial}

Existen otros tipos de metodologías activas que se incluyen en el currículo para fortalecer los 
procesos de enseñanza y favorecer la calidad académica en la ingeniería industrial tales como el aprendizaje basado en proyectos, aprendizaje basado en la indagación, aprendizaje colaborativo, entre otras (Mejía y López, 2017).

Roig-Zamora (2014) aplica el aprendizaje colaborativo para que los estudiantes utilicen los conocimientos del curso de Logística de la cadena de valor I de la Universidad de Costa Rica en entornos complejos, reales y concretos. La metodología sigue tres pasos claves: de actividad, de bidireccionalidad y de colaboración. Los resultados demuestran que la metodología ayuda a facilitar la aplicación de los conocimientos en situaciones reales y escalables a otros problemas de industrias similares. Laguado, Ramírez y Hernández (2019) aplican el aprendizaje basado en proyectos al curso de prácticas industriales para el fortalecimiento de la relación universidad-empresa. Con ello se logra el fortalecimiento de las competencias colaborativas, cognitivas y de investigación de los estudiantes; los proyectos tienen como finalidad problematizar la realidad mediante el estímulo de la interrogación constante frente a las cosas. Se realizan durante un tiempo limitado y generalmente siguen tres fases: preparación, desarrollo y comunicación (LaCueva, 1998).

Por otra parte, la indagación también es empleada para fortalecer el proceso de investigación, generación de conocimientos y apoyo a la educación (Camacho, Casilla y Finol de Franco, 2008). En el trabajo de Lappas y Kritikos (2018) aplican esta metodología en el área de optimización, la cual es de gran importancia en la ingeniería industrial. Se emplean tres pilares con base en el proceso de investigación científica (PoSI), los cuales son: pasos para resolver problemas con algoritmos, construcción de mapas conceptuales y programación informática. En cada paso se apoyan de las TIC gracias a herramientas como MATLAB y CMAP. Los resultados muestran el aumento del potencial de los estudiantes para resolver problemas complejos a través del uso de algoritmos.

\section{Materiales y métodos}

\section{Contextualización del curso}

La eficiencia en la logística como corazón de la cadena de suministros supone un proceso de planeación, organización, seguimiento y control eficiente de todos los bienes y servicios, de tal manera que se puedan entregar en las mejores condiciones de calidad, en el momento exacto, en la cantidad solicitada y en el lugar indicado, todo, al menor costo posible (Reyes, Zavala y Gálvez, 2008); por lo cual representa, dentro de la formación del ingeniero industrial, un campo de gran importancia en del área de diseño de sistemas productivos cuya base es el diseño y la optimización. Ver Figura 2.

En este sentido y en concordancia con la competencia específica del programa, se promueve que el estudiante logre los siguientes desempeños: i. estructurar una cadena de suministros para la

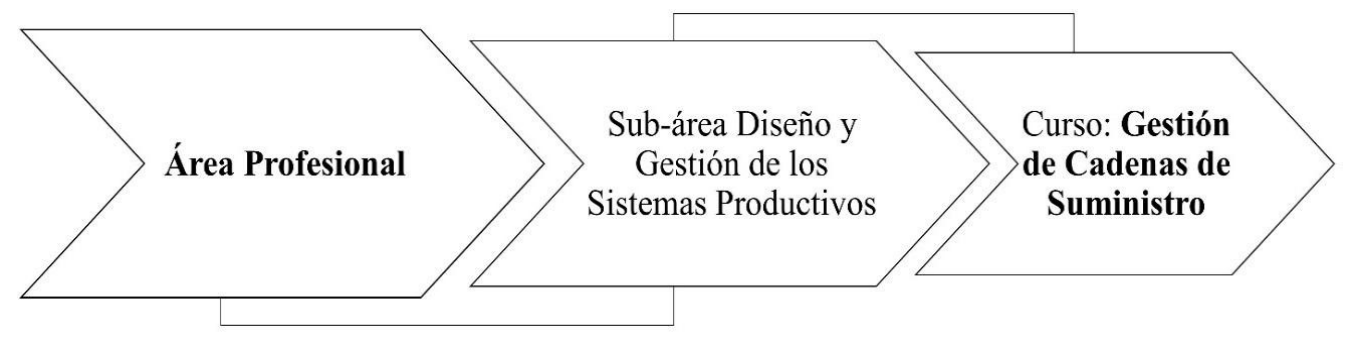

Figura 2. Estructura áreas y sub-áreas que orientan al curso de Gestión de Cadenas de Suministro desde la competencia del programa.

Fuente: elaboración propia a partir de Programa de Ingeniería Industrial (2018). 
identificación de los principales eslabones, actores, roles, actividades logísticas y diagnóstico de problemas a través de un informe del caso problema contextualizado; ii. aplicar modelos de selección de proveedores con base en análisis de pronósticos y criterios de selección a través de un informe del caso aplicado; iii. aplicar sistemas de transporte y transferencia de materiales dentro de los procesos de producción mediante un informe del caso aplicado de estudio; iv. comprender los principales modos y medios de transporte y las plataformas logísticas asociadas, mediante la caracterización de los principales puertos y aeropuertos nacionales e internacionales en un informe de revisión; v. solucionar problemas de ruteo de vehículos en el caso aplicado mediante la resolución de casos problema de modelación matemática y programación lineal entera mixta con apoyo del software de optimización GAMS; vi. desarrollar soluciones de problemas desde el campo de la logística con enfoque en: modelos de transporte, modelos de localización, modelos de simulación y optimización de procesos de producción, herramientas aplicadas al proceso de aprovisionamiento, entre otras, que permitan poner en práctica los temas abordados en el curso mediante un caso problema contextualizado, dando un buen uso a la documentación e información suministrada por la empresa impidiendo o evitando su sustracción, destrucción, ocultamiento o utilización indebida, de conformidad con los fines a que hayan sido destinados.

\section{Metodología de aplicación}

La aplicación del ABP se realizó en los periodos 2018 II y 2019 I. Para el periodo de 2018 II se destinó el grupo denominado B de 23 estudiantes. Para el periodo 2019 I se escogió al grupo denominado C conformado por 31 estudiantes. Se tomó al grupo denominado D conformado por 20 estudiantes como grupo de control (no se aplicó la metodología). Adicionalmente, se compararon con el grupo denominado A del periodo 2018-I, en el cual no se había aplicado la metodología. Todos los grupos eran pertenecientes a IX semestre de Ingeniería Industrial de la Corporación Universitaria del Caribe CECAR, ubicada en el departamento de Sucre, Colombia. La metodología de trabajo se desarrolló en cinco fases que permitieron la construcción de productos finales. Ver Figura 3.

Identificación de situaciones problema contextualizadas

Desde el enfoque de la implementación del ABP, se generan tres panoramas de situaciones problemas partiendo de una realidad del departamento y enfocados en tres empresas en particular, las cuales, por aspectos de confidencialidad e información cifrada, se denominan empresa A, B y C.

Panorama general situación problema empresa $A$ : la red logística de la empresa A presenta una estructura que va desde el abastecimiento de materia prima, la producción de ciertos materiales

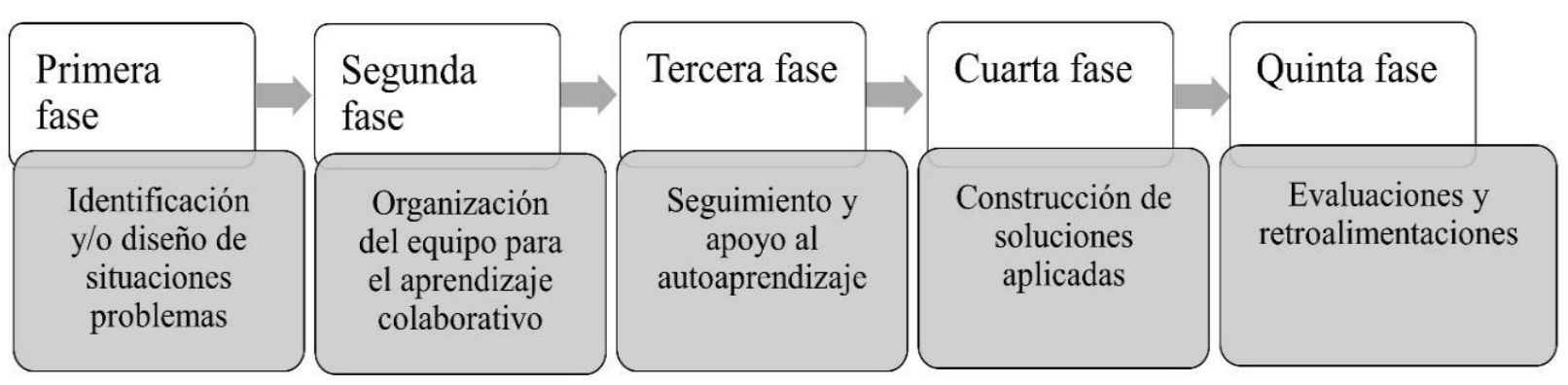

Figura 3. Fases aplicadas en la metodología ABP en Ingeniería Industrial.

Fuente: elaboración propia. 
como bloques vibrados y el proceso de trituración de piedra caliza, hasta la distribución de los productos fabricados. Asimismo, maneja otra gama de productos como arena, cementos, herramientas para la construcción y baterías sanitarias. Sin embargo, esta empresa no tiene una estructura logística definida en cuanto a su articulación dentro de la cadena de suministros a la que pertenece en el departamento de Sucre. Por lo cual es necesario identificar dentro de la cadena departamental o municipal la estructura logística y la participación de la empresa como actor relevante en alguno de los eslabones de la macro cadena.

Adicionalmente, esta empresa cuenta con un actor principal que son los clientes finales con una política de entregas a domicilio, es decir que la empresa transporta los productos hasta el destino del consumidor, lo que implica una logística de distribución para determinar cómo transportar los materiales a los lugares de destino; consideraciones específicas para esta logística son: acumulación de pedidos de diferentes clientes, diferentes tipos de vehículos disponibles en la empresa y su capacidad de carga, horarios de entrega de pedidos, y diferentes ubicaciones de los clientes. Actualmente la empresa toma las decisiones de transporte de manera empírica y dependiendo de la disponibilidad momentánea de su flota de vehículos. Esto genera errores y costos innecesarios que requieren obtener soluciones logísticas de manera que la empresa se articule con la cadena de suministro y mejore sus procesos de distribución.

\section{Panorama general situación problema em-}

presa B: esta empresa se dedica a la fabricación de ladrillos. Los problemas giran en torno a los sobrecostos de producción por el alto índice de productos no conformes que representan aproximadamente el $13 \%$ de la producción total. Adicionalmente, muestra una baja calidad en los productos terminados, debido a la falta de estandarización. Otro problema corresponde a los retrasos en los pedidos de los clientes tanto en fabricación como en entregas. Lo que infiere un problema de producción y distribución de los productos. Estos problemas generan una ruptura o un desequilibrio en la organización logística de la empresa, conllevando a la ineficiencia y la baja competitividad por la no satisfacción de los requerimientos de los clientes.

Panorama general situación problema empresa $C$ : luego de realizar una matriz DOFA a la empresa $C$, se observó que el proceso logístico es totalmente empírico, sin existencia de registros de datos sobre pedidos, inventarios, ventas y distribución; al revisar directamente el proceso de aprovisionamiento de materia prima e insumos para la fabricación de productos (utensilios de aseo), se observa que: no hay planeación para la previsión de materias primas, sino que solo se pide en el momento que hay escasez de materiales, y no existe una planeación de la producción como tampoco registros de inventario (materia prima, productos en proceso y productos terminados). Cabe resaltar que la mayor problemática es el no tener inventario suficiente de materias primas, debido a que el proveedor es externo a la región, lo que conlleva a un tiempo de espera de aproximadamente cinco días desde que se realiza el pedido hasta que llega a la empresa.

Todo esto permite observar que la empresa presenta una mala organización logística de aprovisionamiento y no conoce a todos los demás potenciales actores de la cadena (proveedores y clientes), para aumentar así su competitividad dentro del sector de la industria manufacturera.

\section{Organización del trabajo para el aprendizaje colaborativo}

El aprendizaje colaborativo destaca el valor constructivo de la interacción sociocognitiva y de la coordinación o interacción entre estudiantes. Es una teoría que tiene como eje tres corrientes fundamentales: el socio-constructivismo neo-piagetiano, el enfoque neo-vygotskiano de la intersubjetividad y el modelo de la cognición distribuida (Roselli, 2011). En este sentido, para 
lograr la coordinación entre estudiantes, al igual que Betancourt (2006) y Decker-Lange (2018), se crean grupos de trabajo de dos a cuatro estudiantes. Se equilibró la carga de trabajo sin generar roles específicos para que todos trabajaran uniformemente; en total el curso fue dividido en ocho grupos de trabajo para el periodo 2018-Il y nueve grupos en el periodo 2019-I. Para cada grupo se seleccionó una situación problema específica. El rol del docente pasó a ser solo de un guía de orientación en cuanto a metodologías de trabajo, profundización de conceptos, y asesorías dentro y fuera del aula de clases. Así, se realizó un desarrollo de mayor eficiencia de los conocimientos relevantes y la aplicación de conceptos previos vistos no solo en el curso, sino a lo largo del proceso de formación profesional (Promentilla, Lucas, Aviso y Tan, 2017).

Seguimiento y apoyo al autoaprendizaje

La orientación de la implementación de la didáctica ABP se establece desde el programa de curso y se detallan las estrategias en los syllabus; con lo cual, las clases se estructuran con base en el desarrollo de la didáctica. De igual modo, se construyen las rúbricas de evaluación de cada una de las actividades asociadas a los desempeños en lo concerniente a los criterios de evaluación del desempeño.

Para la realización de las actividades, el estudiante, desde la orientación didáctica de la metodología del ABP, aprende a aprender, a investigar y a trabajar colaborativamente (Betancourt, 2006). En este sentido, los grupos deben dirigirse hasta las empresas A, B y C correspondientes, para observar los problemas mencionados e iniciar mediante una búsqueda de información primaria y secundaria, apoyada en las bases de datos científicas, su articulación en una cadena de suministro global, por lo que paralelamente en clase, y a través de documentos y artículos científicos, el docente suministra información que permite a los estudiantes tener una claridad de los conceptos.

\section{Construcción de soluciones aplicadas}

Con base en la estructura logística que se elabora para las empresas, además de la obtención de datos de gran importancia y las posibles soluciones identificadas para los problemas, los estudiantes inician la construcción de métodos y aplicación de herramientas de modelación, simulación y cálculos para dar una solución eficiente; paralelo a ello, el docente explica y suministra información relevante sobre un compendio de herramientas, metodologías, modelaciones y simulaciones para atender problemas logísticos en las organizaciones. Entre estas herramientas tenemos las recomendadas por Mora y Martiliano (2010), las cuales son: modelos $\mathrm{ABC}$, modelos de pronósticos de demandas, modelos de inventarios y lote económico, herramientas para selección de proveedores, modelo de áreas de almacenamiento, layout y distribución en planta, modelos de indicadores de gestión de almacenamiento y distribución, modelos de localización de centros de acopio/distribución/plantas, modelos de planeación de la producción, modelos de cargue y volumetría de camiones, modelos de transporte y ruteos de vehículos, herramientas para el análisis de ciclo de vida y huella de carbono, entre otras.

\section{Evaluaciones y retroalimentación}

El proceso de evaluación consiste en la revisión de las actividades principales ligadas a los desempeños, teniendo en cuenta las rúbricas específicas de evaluación construidas con base en las rubricas institucionales. La retroalimentación es un proceso fundamental dentro del ABP, debido a que permite al estudiante conocer el impacto de sus aportes, generar estímulos y confianza en él en cuanto a la pertinencia de su proceso de aprendizaje (Instituto Tecnológico y de Estudios Superiores de Monterrey, s.f.).

Por otra parte, es necesario evaluar el proceso realizado como estrategia de mejora continua como se establece en el ciclo PHVA desarrollado 
por Shewhart y aplicado por Deming, que consiste en planear, hacer, verificar y actuar (García, Quispe y Ráez, 2003; Swamidass, 2000). Para esto se desarrolla una encuesta de percepción que consta de 12 preguntas evaluada a través de una escala cualitativa (totalmente de acuerdo, de acuerdo, ni de acuerdo ni desacuerdo, en desacuerdo y totalmente en desacuerdo). La encuesta se diseña a partir de los planteamientos del Instituto Tecnológico y de Estudios Superiores de Monterrey (s.f.) y Dean (2001). Las preguntas que hacen referencia en general a la pertinencia de la metodología y el rol del docente se detallan en la tabla 1.

\section{Resultados}

\section{Construcción de soluciones basadas en las fases del ABP propuestas}

Los resultados obtenidos con la aplicación del ABP en el curso de Gestión de Cadenas de Suministro se reflejan en un compilado de artículos potenciales (nueve en el periodo 2019-I y ocho en el periodo
2018-II) enfocados en simulación y optimización. En ambos semestres se seleccionaron los mejores documentos para participar en el evento institucional de feria del diseño del programa de Ingeniería Industrial. Los resultados de solución aplicados se detallan en la tabla 2.

Por otra parte, se evidencia un aumento en las calificaciones obtenidas de los grupos en los que se aplicó el ABP. En el grupo A no se había aplicado la metodología y se evidencia una mejora en las calificaciones del grupo B. Para los grupos del periodo 2019-I (C y D), se evidencia la diferencia de calificación. Ver Figura 4.

\section{Percepción de los estudiantes}

Para validar el grado de aceptación de la estrategia en el curso, se aplica la encuesta orientada a percepción de los estudiantes en cuanto a la metodología aplicada. Los resultados evidencian que el $45,83 \%$ de los estudiantes está totalmente de acuerdo con la aplicación. El 52,78\% está de acuerdo y, finalmente, el 1,39\% no está ni de acuerdo ni en desacuerdo. Ver Figura 5.

Tabla 1. Encuesta de percepción.

\begin{tabular}{|c|c|}
\hline Número & Afirmaciones \\
\hline 1 & $\begin{array}{c}\text { Determiné las personas implicadas en el problema y sus características, la relación de estas con el problema o situaciones } \\
\text { presentados. }\end{array}$ \\
\hline 2 & Analicé los elementos que se relacionan indirectamente e intervienen en el problema y la teoría que lo sustenta. \\
\hline 3 & $\begin{array}{c}\text { Presenté soluciones al problema o situación presentados por el profesor, de forma clara, coherente y precisas a través de } \\
\text { entregables y sustentaciones. }\end{array}$ \\
\hline 4 & Organizamos las actividades y roles para cumplir con los retos planteados. \\
\hline 5 & Las actividades propuestas fueron apropiadas para el trabajo en equipo y la resolución del problema. \\
\hline 6 & El problema fue definido claramente [por] el profesor además de entregarme información nueva y relevante. \\
\hline 7 & El profesor explicó con claridad la información y actividades a realizar. \\
\hline 8 & $\begin{array}{c}\text { La estrategia propuesta a partir de un problema me incentivó a desarrollar actividades para profundizar y generar nuevos } \\
\text { conocimientos. }\end{array}$ \\
\hline 9 & La estrategia utilizada por el profesor me permitió poder aprender o relacionar el conocimiento en el contexto real. \\
\hline 10 & El profesor propuso preguntas perspicaces que permitieron identificar el problema, formular hipótesis, entre otros. \\
\hline 11 & $\begin{array}{c}\text { La estrategia propuesta por el profesor me permitió fortalecer el trabajo individual como un elemento importante para el aporte } \\
\text { grupal y la solución del problema. }\end{array}$ \\
\hline 12 & $\begin{array}{c}\text { El problema solucionado se resolvió desde diferentes campos disciplinarios, comprendiendo los diferentes factores con los que } \\
\text { se relaciona. }\end{array}$ \\
\hline
\end{tabular}


Tabla 2. Soluciones aplicadas a las empresas A, B y C.

\begin{tabular}{|c|c|c|}
\hline $\mathrm{N}^{\circ}$ & Empresa & Solución \\
\hline 1 & A & $\begin{array}{l}\text { Diseño de un modelo matemático de ruteo de vehículos y comparación de soluciones con la heurística de Clarke \& } \\
\text { Wright con diferentes escenarios. Se obtiene eficiencia en la distribución. }\end{array}$ \\
\hline 2 & A & $\begin{array}{l}\text { Diseño de un modelo de ruteo con variantes de multiproducto capacitado y flota heterogénea para la distribución de } \\
\text { pedidos. Con esta herramienta se evidencia un aumento del } 8.22 \% \text { del beneficio sobre el proceso de distribución. }\end{array}$ \\
\hline 3 & A & $\begin{array}{l}\text { Diseño de un modelo de ruteo capacitado con flota heterogénea para la distribución de pedidos. El modelo fue resuelto } \\
\text { con el solver CPLEX de GAMS mostrando una reducción del } 11.69 \% \text { de los costos actuales. }\end{array}$ \\
\hline 4 & B & $\begin{array}{l}\text { Diseño de un modelo de programación entera mixta para el aprovisionamiento y la planeación de la producción. Se basa } \\
\text { en un pronóstico de demanda con el método ARIMA y un análisis y una selección de proveedores mediante el Proceso de } \\
\text { Análisis Jerárquico (AHP). }\end{array}$ \\
\hline 5 & B & $\begin{array}{l}\text { Diseño de un modelo de planificación de producción y distribución de diferentes tipos de productos seleccionados } \\
\text { mediante un análisis de Pareto. }\end{array}$ \\
\hline 6 & B & $\begin{array}{l}\text { Diseño de un modelo matemático para el proceso de recolección de la materia prima y el esquema de producción para } \\
\text { diferentes tipos de productos en tiempos regulares y extras. Las soluciones evidencian un aumento de la utilidad del } \\
27.88 \% \text { aproximadamente. }\end{array}$ \\
\hline 7 & $\mathrm{C}$ & $\begin{array}{c}\text { Diseño de un modelo de inventarios (EOQ) y simulación en redes de Petri para determinar la cantidad óptima de materias } \\
\text { primas, los tiempos de pedidos y la planificación de la producción. }\end{array}$ \\
\hline
\end{tabular}

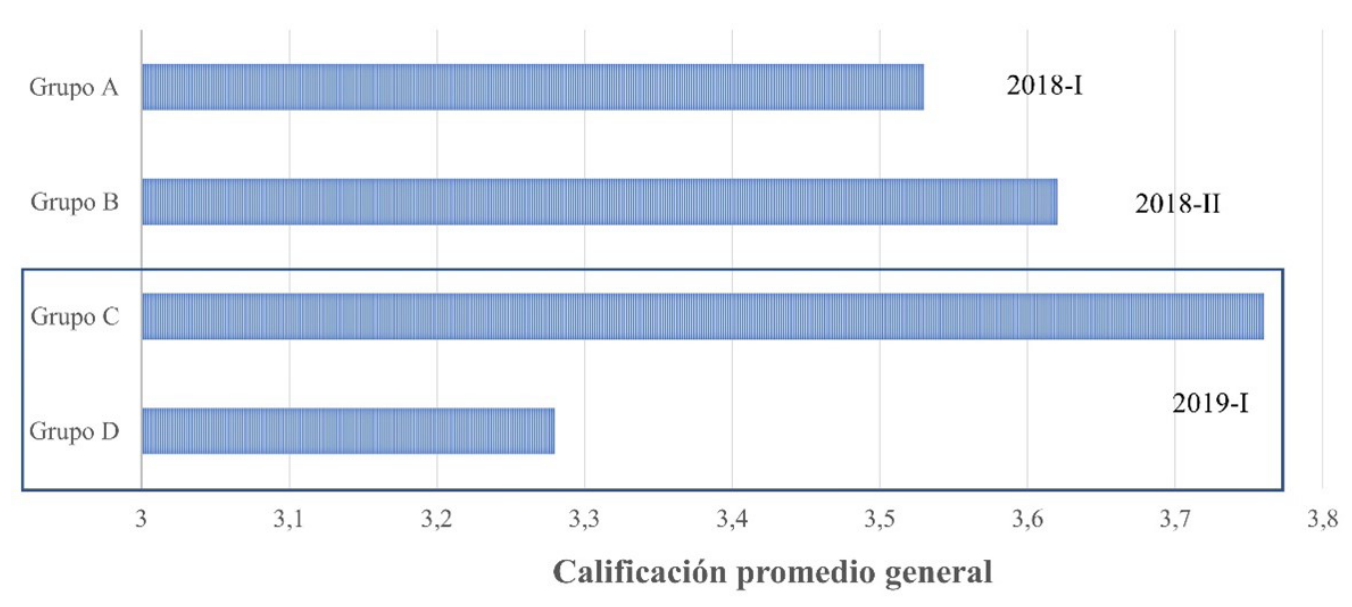

Figura 4. Comparación de notas periodos 2018 I, 2018 II y 2019 I.

Fuente: elaboración propia.

\section{Discusión y conclusiones}

Solucionar problemas sociales, económicos, ambientales y organizacionales del contexto real procurando la eficiencia de los recursos, materiales, herramientas y energía es una de las principales competencias de los ingenieros (Fernández y
Duarte, 2013); por lo cual se deben aplicar metodologías de enseñanza-aprendizaje que procuren el alcance de las competencias teniendo en cuenta el contexto de la región.

La metodología de ABP permite la aplicación de modelos de aprendizaje colaborativo y aprendizaje significativo que se dan en el ejercicio propio 


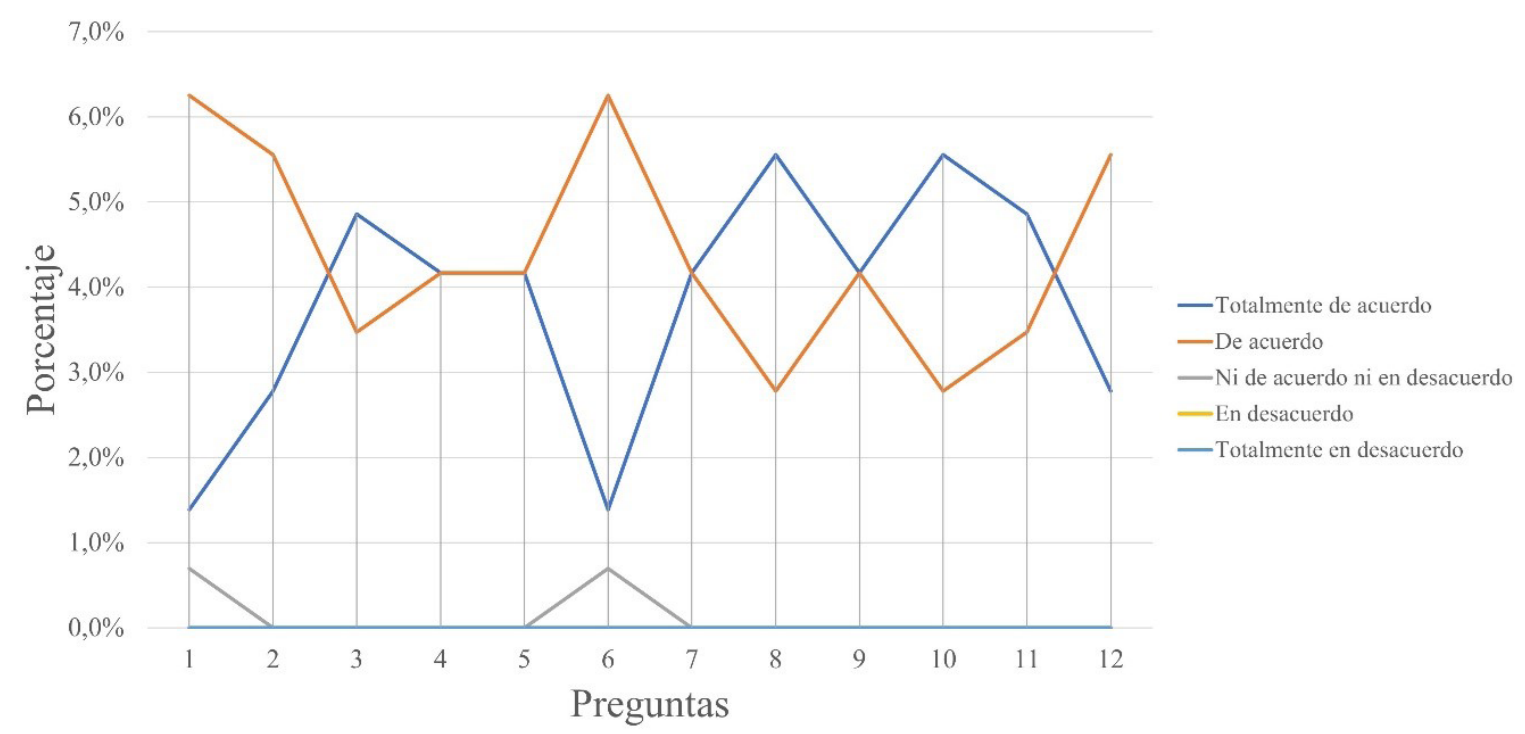

Figura 5. Gráfico de respuestas obtenidas.

Fuente: elaboración propia.

de la indagación que debe realizar el estudiante con su grupo de trabajo, a partir de una situación problema que facilita el docente, orientada a un contexto real. Esto conlleva al docente a convertirse solo en un orientador, un asesor del estudiante, para que él mismo sea capaz de construir su propio conocimiento con base en lo que observa, lo que indaga, sus conocimientos previos y el apoyo del docente. Asimismo, a nivel curricular conlleva al diseño de syllabus estructurados en torno al ejercicio mismo del ABP. Por esta razón, el syllabus del curso referenciado en este trabajo se construyó de tal manera que, además de responder a las teorías constructivistas de la educación y al enfoque por competencias, aplicara el ABP en cinco fases. Cada una de las fases fue implementada con éxito logrando obtener productos finales tangibles, los cuales son un compendio de artículos que detallan las soluciones construidas en referencia a las situaciones problema y los enfoques de solución adoptados.

La revisión de literatura demuestra que la aplicación del ABP en el campo de la ingeniería industrial aún es limitada a pesar de la pluralidad de áreas y campos de acción, tanto administrativos como de producción, calidad, logística, gestión humana, entre otros, que presenta esta profesión (Mosquera, 2002; Ruiz y Vergara, 2018; Zambrano y Alvarado, 2011). Sin embargo, los aportes encontrados exhiben la eficiencia de esta metodología en su aplicación a diferentes cursos y de diferentes niveles. Asimismo, se denotan diferencias en los pasos de aplicación, sin dejar de lado las bases y la finalidad del ABP.

Por otra parte, existen otras metodologías activas que generan buenos resultados y que también han sido aplicadas a los cursos de ingeniería industrial. En todas estas, un criterio base es que el estudiante sea el protagonista de su aprendizaje (Mejía y López, 2017). Con relación al ABP, esta metodología permite articular el horizonte pedagógico, el enfoque socio-formativo y los propósitos institucionales. Con ello ayuda a gestar en los sujetos habilidades y capacidades como la creatividad, la reflexión, el análisis, la criticidad y la toma de decisiones oportunas, para aportar el desarrollo integral y desenvolverse en situaciones reales a partir de los conocimientos adquiridos. Sin embargo, la selección y aplicación de una metodología activa debe atender a los objetivos o propósitos que un 
curso requiera, a la disposición de recursos y al grupo de estudiantes que se dirige. En este sentido, se puede asumir que existen metodologías muy parecidas al ABP que comparten el mismo nivel en la adquisición de conocimiento, habilidades, actitudes y competencias; las cuales conectan al estudiante con proyectos, problemas o retos, desde una realidad, para generar una respuesta idónea para el mundo laboral.

De allí que el ABP se implementa por su flexibilidad de adaptación al curso seleccionado, permitiendo que los estudiantes se vinculen e involucren con la búsqueda de los problemas desde el inicio; se logra un análisis del contexto de varias situaciones encontradas a las que se les da nivel de prioridad y se solucionan, lo cual es adicional a la respuesta de la situación específica que se da con otras metodologías. De igual modo, el ABP ayuda a evaluar y reconocer el proceso de resolución de la problemática a diferencia de otras metodologías que solo evalúan el producto o el resultado final. Esto se logra tomando en consideración el análisis en equipo incluyendo al docente, tal y como sucede en entornos reales. Con la aplicación del ABP se generó investigación con la guía del docente, sin perder la ruta inicial.

Ahora bien, para el caso de estudio se evidencia un aumento del promedio de calificaciones de los grupos $\mathrm{B}$ y $\mathrm{C}$ con respecto al grupo $\mathrm{A}$ en el cual no se aplicó. Incluso, al tomar el grupo D de control del periodo 2019-I en comparación con el otro grupo en el mismo periodo, se evidencia un aumento del promedio de calificaciones.

Cabe resaltar que será común encontrar resistencia al cambio y la consecuente aplicación de clases magistrales por parte de algunos docentes, o la no aceptación de la didáctica por parte de algunos estudiantes. No obstante, la orientación pedagógica de la institución insta tanto al cuerpo docente como a los estudiantes a la adopción del modelo sociocognitivo y es ahí donde herramientas como el ABP generan un gran apoyo al proceso. De igual modo, el docente al encontrarse con este tipo de situaciones con estudiantes que generen "ruido" en el proceso, debe abstenerse de volver a la magistralidad y recordar la importancia del aprendizaje significativo, el logro de las competencias específicas y, además, los productos finales que se alcanzarán.

Para futuros trabajos, la aplicación de este tipo de estudios puede orientarse hacía dos ámbitos. El primero consiste en aplicar otros tipos de estrategias en los cursos y evaluar el impacto de cada una y su comparación con las demás. Como segunda opción, seguir aplicando ABP en otros cursos y evaluando su pertinencia.

\section{Referencias}

Andersen, A.-L., Brunoe, T. D., Nielsen, K. (2019). Engineering Education in Changeable and Reconfigurable Manufacturing: Using Problem-Based Learning in a Learning Factory Environment. Procedia CIRP, 81, 7-12. https://doi.org/10.1016/j. procir.2019.03.002

Barrows, H. S. (1996). Problem-Based Learning in Medicine and Beyond: A Brief Overview. En L. Wilkerson \& W. Gijselaers (Eds.), Theory and Practice. New Directions for Teaching and Learning, 68, 3-12. https://doi.org/10.1002/tl.37219966804

Baykasoğlu, A., Demirkol Akyol, S., \& Demirkan, B. (2017). An Excel-Based Program to Teach Students Quick Ergonomic Risk Assessment Techniques with an Application to an Assembly System. Computer Applications in Engineering Education, 25(3), 489507. https://doi.org/10.1002/cae.21816

Betancourt, C. (2006). Aprendizaje basado en problemas una experiencia novedosa en la enseñanza de la ingeniería. Educación en Ingeniería, 1(2), 45-51.

Camacho, H., Casilla, D., Finol de Franco, M. (2008). La indagación: una estrategia innovadora para el aprendizaje de procesos de investigación. Laurus, 14(26), 284-306.

Dean, C. (2001). They expect teachers to do what? Helping teachers to explore and take ownership of their profession. En B. Levin (Ed.), Energizing Teacher Education and Professional Development with Problem-Based Learning (pp. 8-23). 
Decker-Lange, C. (2018). Problem- and Inquiry-Based Learning in Alternative Contexts: Using Museums in Management Education. The International Journal of Management Education, 16(3), 446-459. https://doi.org/10.1016/j.ijme.2018.08.002

Dolmans, D., Wolfhagen, I., van der Vleuten, C., Wijnen, W. (2001). Solving Problems with Group Work in Problem-based Learning: Hold on to the Philosophy. Medical Education, 35(9), 884-889. https:// doi.org/10.1046/j.1365-2923.2001.00915.x

Echavarria, M. V. (2010). Problem Based Learning Application in Engineering. Escuela de ingenieria de Antioquia, 7, 85-95.

Fernández, F. H., Duarte, J. E. (2013). El aprendizaje basado en problemas como estrategia para el desarrollo de competencias específicas en estudiantes de ingeniería. Formación Universitaria, 6(5), 29-38. https://doi.org/10.4067/S0718-50062013000500005

García, M., Quispe, C., Ráez., L. (2003). Mejora continua de la calidad en los procesos. Industrial Data, 6(1), 89-94.

Herrán, C. A., Vega, C. F. (2006). Uso del ABP como estrategia didáctica para lograr aprendizaje significativo del diseño de ingeniería. Educación En Ingeniería, 1(2), 33-44.

Inelmen, E. (2003). Challenging the Administration to Implement Problem-Based Learning in the Undergraduate Engineering Curriculum. International Journal of Engineering Education, 19(5), 725-729.

Instituto Tecnológico y de Estudios Superiores de Monterrey. (s.f.). El aprendizaje basado en problemas como técnica didáctica. En Las estrategias y técnicas didácticas en el rediseño.

Könings, K. D., Brand-Gruwel, S., van Merriënboer, J. (2005). Towards more Powerful Learning Environments through Combining the Perspectives of Designers, Teachers, and Students. British Journal of Educational Psychology, 75(4), 645-660. https:// doi.org/10.1348/000709905X43616

LaCueva, A. (1998). La enseñanza por proyectos: ¿mito o reto? Revista Iberoamericana de Educación, 16, 165-187. https://doi.org/10.35362/rie1601117

Laguado, R., Ramírez, P., Hernández, F. Y. (2019). El aprendizaje basado en proyectos, una experiencia en las prácticas industriales del Programa de Ingeniería Industrial de la UFPS. Bistua: Revista de la Facultad de Ciencias Básicas, 17(3), 80-89.

Lappas, P., Kritikos, M. (2018). Teaching and Learning Numerical Analysis and Optimization: A Didactic Framework and Applications of Inquiry-Based Learning. Higher Education Studies, 8(1), 42-57. https:// doi.org/10.5539/hes.v8n1p42

Lau, H. Y. K., Mak, K. L. (2004). The Virtual Company: A Re-Configurable Open Shell for Problem-Based Learning in Industrial Engineering. Computers and Industrial Engineering, 47(2-3), pp. 289-312. https://doi.org/10.1016/j.cie.2004.08.002

Lau, H. Y. K., Mak, K. L. (2005). A Configurable E-Learning System for Industrial Engineering. International Journal of Engineering Education, 21(2), 262-276.

Lau, H. Y. K., Mak, K. L., Ma, H. (2006). IMELS: An E-Learning Platform for Industrial Engineering. Computer Applications in Engineering Education, 14(1), 53-63. https://doi.org/10.1002/cae.20067

Martínez-León, H. C. (2019). Bridging Theory and Practice with Lean Six Sigma Capstone Design Projects. Quality Assurance in Education, 27(1), 41-55. https://doi.org/10.1108/QAE-07-2018-0079

Mejía, L. A., López, D. C. (2017). Una mirada a las estrategias y técnicas didácticas en la educación en ingeniería. Entre Ciencia e Ingeniería, 11(21), 123-132.

Mesa, J. M., Álvarez, J. V., Villanueva, J. M., de Cos, F. J. (2008). Actualización de métodos de enseñanza-aprendizaje en asignaturas de dirección de proyectos de ingeniería. Formación Universitaria, 1(4), 23-28. https://doi.org/10.4067/ s0718-50062008000400004

Mincomercio. (2019). Información: Perfiles Económicos Departamentales. Bogotá.

Mora, L. A., Martiliano, M. (2010). Modelos de optimización de la gestión logística. La aplicación de herramientas estadísticas para la planeación y simulación en la cadena de abastecimiento. Ecoe Ediciones.

Mosquera, F. J. (2002). Introducción de la Ingeniería Industrial: un enfoque humanístico. Universidad Industrial de Santander. 
Moust, J. H. C., van Berkel, H. J. M., Schmidt, H. G. (2005). Signs of Erosion: Reflections on Three Decades of Problem-Based Learning at Maastricht University. Higher Education, 50(4), 665-683. https://doi.org/10.1007/s10734-004-6371-z

Muñoz, M., Martínez, C., Cárdenas, C., Cepeda, M. (2013). Active Learning in first-year engineering courses at Universidad Católica de la Santísima Concepción, Chile. Australasian Journal of Engineering Education, 19(1), 27-38.

Nande-Betancurt, J. R. (2016). Una alternativa para favorecer el aprendizaje en ingeniería con la aplicación del aprendizaje cognitivo. Revista Cubana de Educación Superior, 35(2), 104-114.

Ostojic, G., Stankovski, S., Tarjan, L., Senk, I., Jovanovic, V. (2010). Development and Implementation of Didactic Sets in Mechatronics and Industrial Engineering Courses. International Journal of Engineering Education, 26(1), 2-8.

Parra, J. E., Amariles, M. J. , Castro, C. A. (2016). Aprendizaje basado en problemas en el camino a la innovación en ingeniería. Ingenierías USBMed, 7(2). https://doi.org/10.21500/20275846.2486

Programa de Ingeniería Industrial. (2018). Proyecto Educativo del Programa - PEP de Ingeniería Industrial de CECAR. Sincelejo, Sucre.

Promentilla, M. A., Lucas, R. I., Aviso, K., Tan, R. (2017). Problem-Based Learning of Process Systems Engineering and Process Integration Concepts with Metacognitive Strategies: The Case of P-graphs for Polygeneration Systems. Applied Thermal Engineering, 127, 1317-1325. https://doi.org/10.1016/j. applthermaleng.2017.08.086

Reyes, V., Zavala, D., Gálvez, J. (2008). Una revisión del proceso de la logística inversa y su relación con la logística verde. Ingeniería Industrial, 7(2), 85-98.

Roig-Zamora, J. (2014). Implementación del aprendizaje cooperativo como estrategia didáctica para la construcción del conocimiento, en el área de la logística, en estudiantes de la carrera de ingeniería industrial. Educación, 38(1), 85-99. https://doi. org/10.15517/revedu.v38i1.14379

Roselli, N. (2011). Teoría del aprendizaje colaborativo y teoría de la representación social: convergencias y posibles articulaciones. Revista Colombiana de Ciencias Sociales, 2(2), 173-191.

Rovers, S., Clarebout, G., Savelberg, H., van Merriënboer, J. (2018). Improving Student Expectations of Learning in a Problem-Based Environment. Computers in Human Behavior, 87, 416-423. https://doi. org/10.1016/j.chb.2018.02.016

Ruiz, J., Vergara, C. J. (2018). Historia de la ingeniería industrial. En L. Gomez Cáceres y C. J. Vergara (Eds.), Enfoques, teorías y perspectivas de la ingeniería industrial y sus programas académicos. https://doi.org/10.21892/9789588557694

Soares, F. O., Sepúlveda, M. J., Monteiro, S., Lima, R. M., Dinis-Carvalho, J. (2013). An Integrated Project of Entrepreneurship and Innovation in Engineering Education. Mechatronics, 23(8), 987-996. https:// doi.org/10.1016/j.mechatronics.2012.08.005

Swamidass, P. M. (Ed.). (2000). Deming cycle (PDCA). Encyclopedia of Production and Manufacturing Management. https://doi. org/10.1007/1-4020-0612-8_229

Tortorella, G., Cauchick-Miguel, P. A. (2017). An Initiative for Integrating Problem-Based Learning into a Lean Manufacturing Course of an Industrial Engineering Graduate Program. Producao, 27. https:// doi.org/10.1590/0103-6513.224716

Wedel, A., Müller, C. R., Pfetsch, J., Ittel, A. (2019). Training Teachers' Diagnostic Competence with Problem-based Learning: A Pilot and Replication Study. Teaching and Teacher Education, 86(1), e102909. https://doi.org/10.1016/j.tate.2019.102909

Zambrano, S. M., Alvarado, F. E. (2011). Surgimiento y evolución de la ingeniería industrial. In Vestigium Ire, 4(1),19-28. 